\title{
Comunicação
}

[Communication]

\section{Atividade anti-helmíntica de plantas em frangos de corte naturalmente infectados com Ascaridia galli}

[Anthelminthic activity of plants in broiler chickens naturally infected with Ascaridia galli]

\author{
R.M. Fernandes ${ }^{1}$, M.L.A. Rodrigues ${ }^{2}$, H.R. Borba ${ }^{3}$, M.Z.L.C.M. Fernandes ${ }^{4}$, A. Amorim ${ }^{5}$ \\ ${ }^{1}$ Universidade Federal do Piauí - Centro de Ciências Agrárias \\ Depto. Morfofisiologia Veterinária \\ Campus Agrário da Socopo, s/n \\ 64049-550 - Teresina, PI \\ ${ }^{2}$ Depto. de Parasitologia Animal - IV - UFRRJ \\ ${ }^{3}$ Depto. de Biologia Animal - IB - UFRRJ \\ ${ }^{4}$ Doutoranda do Curso de Ciências Veterinárias da UFRRJ \\ ${ }^{5}$ Universidade Federal Rural do Rio de Janeiro
}

A avicultura vem concentrando esforços em pesquisas nas áreas de genética, nutrição, sanidade e manejo. Porém, o aumento no uso de antibióticos, anticoccidianos, promotores de crescimento e anti-helmínticos tem levado a população a optar por alimentação mais saudável, buscando alimentos produzidos de forma orgânica sem o risco de contaminação por resíduos. No entanto, o retorno à criação de frangos tendo acesso a pasto traz um outro problema, que é o aumento das helmintíases aviárias, pois os animais têm acesso às formas infectantes de diversos helmintos, fechando, assim, o ciclo dos parasitos (Thamsborg et al., 1999).

Permin et al. (1999) encontraram prevalência de $63,8 \%$ e $5 \%$ de Ascaridia galli em aves criadas no sistema extensivo (em campo) e em sistema intensivo (em gaiolas), respectivamente. Esses resultados confirmam o risco de infecção mais acentuada no sistema em campo. Dessa forma, as plantas consideradas na medicina popular como anti-helmínticas oferecem uma alternativa de tratamento para as criações orgânicas, em que o uso de quimioterápicos é restrito.
Em conformidade com Amorim e Borba (1990), são raras as descrições de helmintos sensíveis às dezenas de espécies de plantas medicinais tidas como anti-helmínticas. Assim, este trabalho teve como objetivo avaliar a atividade anti-helmíntica do alho (Allium sativum), da romã (Punica granatum), do cipó-cravo (Tynnanthus labiatus) e do coco-da-baía (Cocos nucífera), plantas mencionadas na medicina popular e na literatura (Pio Corrêa, 1996).

Utilizaram-se 70 frangos, machos e fêmeas, linhagem Hubbard, peso médio $3.300 \mathrm{~g}$, com aproximadamente três meses de idade, infectados naturalmente com A. galli. Foram constituídos seis grupos: grupo $1(\mathrm{n}=20)$ - controle-negativo; grupo $2(\mathrm{n}=10)$-tratado com mebendazole na dose de $0,031 \mathrm{~g} / \mathrm{kg} / \mathrm{dia}$; grupos $3,4,5$ e 6 $(\mathrm{n}=4 \times 10)$ - tratados, respectivamente, com suco de alho a $10 \%$, cipó-cravo (caule triturado), romã (epicarpo triturado) e leite-de-coco (integral) administrados por sonda ou incorporados à ração, nas doses de $2,3,3$ e $10 / \mathrm{kg} / \mathrm{dia}$, respectivamente, durante três dias.

Recebido para publicação em 3 de novembro de 2003 
As aves foram mantidas individualmente em gaiolas de arame galvanizado com fundo removível para facilitar a colheita diária das fezes. Antes do início do experimento, passaram por um período de adaptação de 72 horas, quando receberam, diariamente, $100 \mathrm{~g}$ de ração e água à vontade. Todos os animais foram submetidos a jejum de 18 horas, com água à vontade. As fezes foram coletadas, durante quatro dias, e processadas de acordo com Amato et al. (1991). No quinto dia, os animais foram sacrificados e necropsiados. A eficácia do tratamento foi avaliada pelo método crítico controlado (Steward, 1955), adaptado a este modelo experimental. Os resultados foram expressos em percentuais médios de nematóides eliminados nas fezes em relação ao total de helmintos recuperados na contagem fecal e à necropsia. Usou-se o teste não paramétrico para comparação das médias por meio de valores de
"U" (Whitney, 1975), tanto para os grupos tratados como para o grupo-controle.

Quanto à eliminação de $A$. galli, não houve diferença entre o grupo tratado com suco de alho e o não tratado (Tab. 1), resultado já observado por Peña et al. (1988). O percentual de eliminação no grupo tratado com romã foi menor do que no grupo-controle, resultado discrepante em relação ao expressivo efeito anti-helmíntico observado por Amorim e Borba (1990), em estudos realizados em camundongos portadores de infecção natural de Vampirolepis nana e oxiurídeos. É provável que essa diferença esteja relacionada com a forma de administração, pois neste trabalho empregou-se o epicarpo adicionado à ração. Os resultados sugerem que o princípio ativo relacionado a essa atividade poderá estar na fração aquosa.

Tabela 1. Eficácia do alho, do cipó-cravo, do coco-da-baía, da romã e do mebendazole em frangos naturalmente infectados com Ascaridia galli

\begin{tabular}{|c|c|c|c|c|c|}
\hline Tratamento & $\begin{array}{l}\text { Número de } \\
\text { animais }\end{array}$ & $\begin{array}{c}\text { Número }^{2} \text { de } A \\
\text { galli }\end{array}$ & $\begin{array}{l}\text { Contagem de helmintos à } \\
\text { necropsia (mín./máx.) }\end{array}$ & $\begin{array}{c}\text { Total de } \\
\text { helmintos }\end{array}$ & $\begin{array}{c}\text { Eliminação fecal } \\
(\%)\end{array}$ \\
\hline Não tratado & 20 & 23 & $215(0-80)$ & 238 & 9,70 \\
\hline Alho & 10 & 7 & $65(0-32)$ & 72 & 9,70 \\
\hline Cipó-cravo & 10 & 8 & $40(0-23)$ & 48 & 16,70 \\
\hline Coco-da-baía & 10 & 22 & $94(1-30)$ & 116 & 19,00 \\
\hline Romã & 10 & 9 & $127(1-25)$ & 136 & 6,60 \\
\hline Mebendazole & 10 & 154 & $2(0-2)$ & 156 & $99,00 *$ \\
\hline
\end{tabular}

$* \mathrm{P}<0,05$

Para o cipó-cravo, embora o efeito tenha sido aproximadamente seis vezes inferior ao mebendazole, a percentagem de eliminação de $A$. galli foi maior quando comparada com o grupocontrole (Tab. 1). São inferiores aos resultados obtidos por Amorim et al. (1991), em testes conduzidos em camundongos naturalmente infectados com nematóides oxiurídeos, cuja eficácia foi em torno de $40 \%$.

A administração do leite-de-coco foi capaz de eliminar aproximadamente $19 \%$, resultado superior à eliminação espontânea do parasita, correspondendo a aproximadamente um quinto do resultado do mebendazole, sendo, dentre as plantas testadas, a que apresentou o melhor resultado. Porém, esses foram inferiores aos relatados em camundongos naturalmente infectados com oxiurídeos (Amorim e Borba, 1995). Assim, este estudo permitiu observar a ausência de atividade anti-helmíntica para todas as plantas testadas.

Palavras-chave: frango, plantas, atividade antihelmíntica, Ascaridia galli

\begin{abstract}
The anthelminthic activity of four plants - Allium sativum (garlic), Punica granatum (pomegranate), Tynnanthus labiatus (liana-carnation) and Cocus nucifera (coconut) with the activity of mebendazole was compared. Seventy Hubbard chickens, naturally infected with Ascarídia galli, divided in 5 groups of
\end{abstract}


10 chichens plus a control group (not treated, $n=20$ ) were used in the experiment. The vegetable matter was used in the forms of aqueous extract, juice and triturated, administered by probe or incorporated to the diet, in the doses of 2,3 and $10 \mathrm{~g} / \mathrm{kg} /$ day, for three days. A non parametric test was used to evaluate the anthelminthic effect of the plants. The eliminations of A. galli for the garlic, pomegranate, lianacarnation, coconut and mebendazole were: 9.7; 6.6, 16.7; 19.0 and 99.0\%, respectively. The results showed that those plants do not have anthelminthic activity.

Keywords: chicken, plants, anthelminthic activity, Ascaridia galli

\section{REFERÊNCIAS BIBLIOGRÁFICAS}

AMATO, J.F.R.; BOEGER, W.A.; AMATO, S.B. Protocolos para laboratório: coleta e processamento de parasitos de pescado. Rio de Janeiro: Imprensa Universitária, 1991. 77p.

AMORIM, A.; BORBA, H.R. Ação antihelmíntica de plantas III. Efeito de extrato aquoso de Punica granatum L. (romã) na eliminação de Vampirolepis nana e de oxiurideos em camundongos. Rev. Bras. Farm., v.71, p.8587, 1990.

AMORIM, A.; BORBA, H.R. Ação antihelmíntica de Plantas XI. Influência de extratos brutos de Cocos nucifera L. (Palmae) na eliminação de Vampirolepis nana em camundongos. Rev. Bras. Farm., v.76, p.98-99, 1995.

AMORIM, A.; BORBA, H.R.; AMANO, L.M.. Ação anti-helmíntica de plantas IV. Influência da casca do caule do cipó-cravo (Tynnanthus fasciculatus, Miers, Bignoniaceae) na eliminação de Vampirolepis nana e oxiurídeos em camundongos. Rev. Bras. Farm., v.72, p.92-94, 1991.
PEÑA, N.; AURÓ, A.; SUMANO, H. A comparative trial of garlic its extract and ammonium-potassium tartarate as anthelmintics in carp. J. Ethnopharmacol., v. 24, p.199-203, 1988.

PERMIN, A. BISGAARD, M; FRANDSEN, F. et al. Prevalency of gastrointestinal helminths in diferent poultry production systems. Br. Poult. Sci., v.40, p.439-443, 1999.

CORRÊA, M.P. Dicionário de plantas úteis do Brasil e das exóticas cultivadas. Rio de Janeiro: Min. da Agricultura. IBDF, 1996. 6v.777p.

STEWARD, J.S. Anthelmintic studies: I. A controlled critical entero-nemacidal test. Parasitology, v.45, p.231-241, 1955.

THAMSBORG, S. M.; ROEPSTORFF; LARSEN, M. Integrated and biological control of parasites in organic and convencional production systems. Vet. Parasitol., v.84, p.169186, 1999.

WHITNEY, M. O caso de duas amostras independentes, p.131-144. In: SIEGEL, S. (Ed.). Estatística não paramétrica. São Paulo: McGraw-Hill do Brasil, 1975. 350p. 